\title{
Deductions from the Quaternion Form of Maxwell's Electromagnetic Equations
}

\author{
Jeremy Dunning-Davies ${ }^{1,2}$, Richard Lawrence Norman ${ }^{3}$ \\ ${ }^{1}$ Departments of Mathematics and Physics (retd), University of Hull, Kingston upon Hull, England \\ ${ }^{2}$ Institute for Basic Research, Palm Harbor, FL, USA \\ ${ }^{3}$ Thunder Energies Corporation, Palm Harbor, FL, USA \\ Email: masjd@masjd.karoo.co.uk, editor@thejournalofunconsciouspsychology.com
}

How to cite this paper: Dunning-Davies, J. and Norman, R.L. (2020) Deductions from the Quaternion Form of Maxwell's Electromagnetic Equations. Journal of Modern Physics, 11, 1361-1371.

https://doi.org/10.4236/jmp.2020.119085

Received: August 20, 2020

Accepted: September 19, 2020

Published: September 22, 2020

Copyright $\odot 2020$ by author(s) and Scientific Research Publishing Inc. This work is licensed under the Creative Commons Attribution International License (CC BY 4.0).

http://creativecommons.org/licenses/by/4.0/

\begin{abstract}
It is often claimed that Maxwell's electromagnetic equations were originally written in terms of quaternions. Once returned to that form and treated with left and right hand operators as in the mathematics of P. M. Jack, a new seventh scalar electromagnetic field component emerges with possible relations to clean energy extraction and gravitation. It is the purpose here to examine this approach afresh and see how it might link up with other fairly recent, but little known, work in the field. Again, as with the usual form of Maxwell's equations, a new scalar wave equation is derived but, on this occasion, due to the presence of the scalar component of the quaternion, that equation exhibits a wave speed greater than the speed of light. Historical and present uses within military and humanitarian contexts are considered briefly.
\end{abstract}

\section{Keywords}

Electromagnetic Field, Quaternion, Gravity, Maxwell, Energy, Scalar Wave

\section{Introduction}

Electromagnetic theory has been without a crucial causal element which is to be revealed upon analysis and subsequent treatment of the quaternion form of Maxwell's equations as offered in his Treatise on Electricity and Magnetism [1]. That particular treatment of those quaternion equations of Maxwell has purportedly been found and engineered by government agencies [2] [3] [4] [5]. However, in at least many editions of the said Maxwell text, reference is restricted to the vector part of each quaternion equation only; the extra scalar term simply does not appear and it is this term, quoted so often in some literature [3] [4], which, if in- 
cluded, can lead to some interesting and possibly useful results. The quaternion treatment by Jack of the equations of Maxwell appears to exhibit the same qualities as those claimed in related literature, revealing a new scalar seventh part within the electromagnetic expressions [6]. The potential implications from the inclusion of the new scalar seventh part of the electromagnetic field within the axiomatic basis of physics include both clean energies and possibly even a link with gravitation.

Over the years, many attempts have been made to link electromagnetism and gravity and many seemingly outrageous claims have been made [3] [4]. The purpose of the present investigation is to examine thoroughly the theoretical basis for these claims and to see just how well they, or at least some of them, stand up to careful scrutiny. As a secondary aim, it is hoped to bring the earlier work more into the public domain so that it may be properly assessed by the wider scientific community. Some of this earlier work is quite old, dating back to the beginning of the last century but, given that it was due to the well-known, eminent mathematician Edmund Whittaker, it is all the more surprising that it isn't already clearly in that public domain. It goes without saying that some of the crucial results of Whittaker will be highlighted here, as will some results due to another mathematician of a slightly later date, Kenneth Thornhill.

It was shown some years ago [5] that the popular form of Maxwell's electromagnetic equations could lead to a new scalar wave equation. Here the work of that article is extended and, with the introduction of the scalar component of the quaternion, a new scalar wave equation is produced which crucially exhibits a wave speed in excess of the speed of light. It would be easy to dismiss this result immediately but, given the careful mathematical build-up to its production, it seems more appropriate to consider if it could have a definite physical interpretation. Some comments are made addressing this but, again, this is something for much wider open-minded deliberation. As is seen from a remark by Wallace Thornhill later in this text, the idea of speeds greater than that of light should not be dismissed lightly and, in this context, it should always be remembered that Einstein's assumption which helped lead to the development of special relativity was that the speed of light in a vacuum should remain constant. Indeed, it is well-known that the speed of light in various media does vary from the value in a vacuum so for a wave speed in excess of that for light to appear in a wave equation should not be a cause for alarm but one for which the exact interpretation remains to be found.

\section{History and Context}

There has been much speculation over a period of time that traditional science was losing out by not considering the quaternion form of Maxwell's electromagnetic equations. However, for most, this form of the equations has either been unknown or has not been readily available for scrutiny. Relatively recently, this latter objection has been rectified by publications due to Peter Jack [6] [7]. 
In this cited article [6] he shows how to write Maxwell's electromagnetic equations in the notation of Hamilton's quaternions. This work opens up a whole new area for serious investigation and, as will be seen, leads to some unusual, possibly disquieting, results that could be related to other work concerning scalar waves [5].

Within the evolution of J. C Maxwell's thinking there is mention in earlier work of gravitation emerging from an energetic reduction in the medium present between massive bodies [8], although the nature of the exact mechanisms involved eluded Maxwell as did the notion of negative energy. His work was based entirely on the presence of an aetherial medium mediating action at a distance. Such an all pervasive medium he concluded, had enormous intrinsic energies. Within that same little known volume by Maxwell, caution is urged from the beginning that the new mathematics and hypothetical theory presented are just that, save for two points which are actual demonstrated physical mechanisms of causality; those two mechanism being the aspects of the electromagnetic field responsible for its energy: 1) the magnetic polarizations and 2) the electric polarizations. Movement is associated with point one, tension or strain in an elastic medium with point two. In later work [1] the idea of tension in an electromagnetic medium is further elaborated (chapter XI) as is the aetherial theory of action at a distance (chapter XXIII) and his many fundamental electromagnetic equations are fruitfully presented in the form of quaternions (sections 618-619 quaternion expressions of the electromagnetic equations, and throughout). Those many equations written in quaternion form by Maxwell were then reduced after Maxwell's death by Heaviside to those very few and more manageable equations we now refer to as "Maxwell's equations," which are written in modern vector notation following the works of Gibbs and Heaviside [3].

Working designs extracting energy from the limitless gravitational store have been built and used under military concealment from World War Two onward including working gravity based designs based in the science of T. Brown that have been practically utilized [9] (and references therein). These designs utilize proton and electron plasmas to create gravitational effects. Those government designs appear to use plasma associated scalar waves to move objects and collect clean energies [9]. Government papers and related books state quite plainly that electromagnetic theory inclusive of a new scalar component is indeed being used to gain these effects and, that this same science has been weaponized, and also utilized to gain clean energies as well as biological effects [2] [3] [4] [5] [9] [10] [11] [12]. That new EM field component is associated with positive and negative time, gravity, positive and negative energies, temperature and charge. Positive time and energies are associated with negatively charged electron interactions and heating; negative time and energies are associated with positive charge, proton interactions and cooling. Those gravitational time associated energies can also be extracted directly [10] [11] [12]. It is stated quite strenuously that the missing scalar field component is derived from Maxwell's original quaternion mathematics [3]. The working quaternion mathematics in question is omitted, 
presumably for purposes of weapons' design secrecy [2]. This leaves the bulk of humanity without the knowledge needed to rightly sustain itself.

It might be noted at this point that any such gravitational expression must propagate at superluminal speeds to maintain contact with celestial mechanics [9] [13].

It is not immediately obvious how the quaternion EM equations of Maxwell contain the missing information related to these temperature, charge, time and gravitational effects [1]. A clever interpretation which introduces many fundamental differences including right-hand and left-hand derivatives devised by a mathematician named Peter Michael Jack appears to have captured the facts correctly [6] [7]. In this insightful work we find the actual mathematics in question, which do show a seventh scalar part to the electromagnetic field:-the Temporal Field $T$. This new field appears to be directly related to the basic scalar wave equation offered up as a solution in CIA work [5]. The Temporal Field $T$ also offers the correct charge related thermal capacities of heat addition in positive work, and also heat subtraction in negative work said to be utilized in weaponized applications, and the negative energetic aspects said to be functioning in free energy devices [2] [12].

Also it seems possible that these mathematics of Jack [6] [7], or some like them, are those referred to in prioritized military applications and could well include gravitational, energetic and temporal qualities as have been ascribed to scalar waves in CIA work and the above referenced and related texts, as well as in our theories.

This mathematics may now be utilized to calculate potential wave propagation speeds emerging from Temporal Field expressions. Is such a wave or is it not superluminal? Note that the needed $T$ field pulsed expression [6] is indeed characteristic of a gravity wave [13]. Before performing those calculations, the relevant literature must be followed, some more history understood and the wave forms' general type derived as being longitudinal in nature.

In relevant literature [3] [4] [13] [14] [15] it is stressed that the work of Whittaker is fundamental in the understanding of these ideas as they are related to gravity and the scalar potentials.

Note that in that same government literature, just as in the work of Maxwell and Whittaker, an ether is given fundamental prominence. "The concept of an ether is again accepted." [3] (p.139)

From Sweet and Bearden:-Utilizing Scalar Electromagnetics To Tap Vacuum Energy [11]

-Based on E.T. Whittaker's previously unnoticed 1903-1904 papers which established a hidden bidirectional electromagnetic wave structure in a standing force field free scalar potential, a method of directly engineering the ambient potential of the vacuum has been developed and realized experimentally.

... Several models have been built, ranging from 6 watts early on to one of 5 kilowatts. Both closed battery-less systems with damped positive feedback and open loop systems with battery-powered input have been successfully built. 
Open loop power gains of from $5 \times 10^{4}$ to $1.5 \times 10^{6}$ have been achieved. Antigravity experiments have also been successfully conducted where the weight of the unit was reduced by $90 \%$ in controlled experiments, with a signal wave input of 175 microwatts and an output of 1 kilowatt."

\subsection{Whittaker and Longitudinal Gravitational Waves}

The name of Edmund Whittaker is familiar to many. Countless students have learned much from the book he co-authored with Watson on Modern Analysis [16] while his two volume work on a history of the theories of Aether and Electricity [17] has proved of inestimable value to many scientists both as a reference text and as a source of inspiration for researchers and historians of science alike. However, although obviously a well-known and well-respected member of the academic community, some of his important papers have seemingly been ignored or carefully forgotten. Among these is his wonderfully detailed work on solutions of partial differential equations of mathematical physics [15], a piece all the more worthy of investigation given the author's extensive knowledge of the topic as evidenced by his aforementioned book on modern analysis. Although the entire paper is, or should be, of interest to all, it is the final section in which he considers the explanation of gravitation and electrostatic attraction as modes of wave disturbance which is of the utmost relevance here. Earlier in the article he has shown that any solution of the equation

$$
\frac{\partial^{2} V}{\partial x^{2}}+\frac{\partial^{2} V}{\partial y^{2}}+\frac{\partial^{2} V}{\partial z^{2}}=k^{2} \frac{\partial^{2} V}{\partial t^{2}}
$$

may be analysed into simple plane waves. He goes on to point out that this throws new light on forces, such as gravitation, which vary via an inverse square law. This is due to the fact that such forces have a potential which satisfies the equation

$$
\frac{\partial^{2} V}{\partial x^{2}}+\frac{\partial^{2} V}{\partial y^{2}}+\frac{\partial^{2} V}{\partial z^{2}}=0
$$

and must, therefore, satisfy Equation (1), where $k$ is any constant. He goes on to point out that this potential may be analysed into simple plane waves. He then proceeds to comment that it is not difficult to construct systems of coexistent simple waves possessing the property that the total disturbance at any point varies from point to point but does not vary with time. He illustrates this by considering a particle emitting spherical waves such that the disturbance at a distance $r$ from the origin at time $t$ due to those waves whose wave-lengths lie between $2 \pi / \mu$ and $2 \pi /(\mu+\mathrm{d} \mu)$ is represented by

$$
\frac{2 \mathrm{~d} \mu}{\pi \mu} \frac{\sin (\mu V t-\mu r)}{r}
$$

where $V$ is the velocity of propagation of the waves. After the waves have reached the point $r$, so that $(V t-r)$ is positive, the total disturbance at this point is given by 


$$
\int_{0}^{\infty} \frac{2 \mathrm{~d} \mu}{\pi \mu} \frac{\sin (\mu V t-\mu r)}{r}
$$

Using the substitution $y=\mu V t-\mu r$, the value of this integral is easily seen to be $1 / r$, that is, the total disturbance at any point, due to this system of waves, is independent of time and is proportional everywhere to the gravitational potential due to the particle at the point.

Whittaker continues by making it clear that this indicates that the field of force due to a gravitating body may be analysed into an infinite number of constituent fields and, although the whole field of force does not vary with time, each of the constituent fields has an undulatory character consisting of a simple wave disturbance propagated with uniform velocity. He further points out that analysis of the field into constituent fields is most easily accomplished by analysing the potential $1 / r$ of each attracting particle into terms of the type

$$
\frac{\sin (\mu V t-\mu r)}{r}
$$

as in the example above. One of the constituent fields will correspond to each of these terms. In each of the constituent fields the potential will be constant along each wave front and consequently the gravitational force in each constituent field will be perpendicular to the wave front-that is, the waves will be longitudinal.

He goes on to draw attention to the fact that "these results assimilate the propagation of gravity to that of light; for the undulatory phenomena just described, in which the varying vector is a gravitational force perpendicular to the wave-front, may be compared with the undulatory phenomena made familiar by the electromagnetic theory of light, in which the varying vectors consist of electric and magnetic forces parallel to the wave-front. The waves are in other respects exactly similar, and it seems probable that an identical property of the medium ensures their transmission through space. This undulatory theory of gravity would require that gravity should be propagated with a finite velocity, which however need not be the same as that of light, and may be enormously greater." It must be stressed, as Whittaker himself does, that this work does not claim to explain the cause of gravity; all it does is show that in order to account for the propagation through space of forces which vary as the inverse square of the distance, it is necessary only to suppose the medium capable of transmitting, with a definite, if large, velocity, simple periodic undulatory disturbances, similar to those whose propagation by the medium constitutes the transmission of light according to the electromagnetic theory.

\subsection{Jack's Analysis}

In his article, Jack takes as his starting point the assumption that the electromagnetic potential may be written as a quaternion

$$
A=\phi+A_{1} i+A_{2} j+A_{3} k,
$$


where $\phi$ is the usual scalar potential and $A_{1}, A_{2}, A_{3}$ are the three components of the vector potential denoted, as usual, by $A$ and $(\mathrm{d} / \mathrm{d} r)$ is the differential operator defined by

$$
\frac{\mathrm{d}}{\mathrm{d} r}=\frac{1}{c} \frac{\partial}{\partial t}+\frac{\partial}{\partial x}+\frac{\partial}{\partial y}+\frac{\partial}{\partial z} .
$$

Note that the non-bold $A$, as distinct from the bold $A$, denotes the quaternion which Jack takes as his starting point.

Then, purely by inspection, the electric and magnetic fields as quaternions are seen to be given by

$$
\begin{aligned}
E & =-\left\{\frac{\mathrm{d}}{\mathrm{d} r}, A\right\}=-(1 / 2)(\mathrm{d} / \mathrm{d} r \rightarrow A+A \rightarrow \mathrm{d} / \mathrm{d} r) \\
& =-\frac{1}{c} \frac{\partial \phi}{\partial t}+\nabla \cdot \boldsymbol{A}-\frac{1}{c} \frac{\partial A}{\partial t}-\nabla \phi
\end{aligned}
$$

and

$$
B=+[\mathrm{d} / \mathrm{d} r, A]=+(1 / 2)(\mathrm{d} / \mathrm{d} r \rightarrow A-A \rightarrow \mathrm{d} / \mathrm{d} r)=\nabla \times \boldsymbol{A} .
$$

Here there has been alternation between the more commonly used 3-vector notion and the 3-vector of Hamilton's quaternion notation, always taking care to match up the components of the appropriate expressions. It might be noted that the space components of these quaternion fields correspond exactly to the usual electric and magnetic fields encountered using the normal 3-vector calculus. However, the quaternion electric field has an extra-time component which is a scalar that may be denoted by $T$ and given by

$$
T=-\frac{1}{c} \frac{\partial \phi}{\partial t}+\nabla \cdot A
$$

This leaves the usual expressions for the electric $(\boldsymbol{E})$ and magnetic $(\boldsymbol{B})$ fields in terms of the usual scalar and vector potentials:-

$$
\boldsymbol{E}=-\nabla \phi-\frac{1}{c} \frac{\partial \boldsymbol{A}}{\partial t} \text { and } \boldsymbol{B}=\nabla \times \boldsymbol{A}
$$

Again purely by inspection, the reformulated Maxwell Electromagnetic Field equations are seen to follow from

$$
[\mathrm{d} / \mathrm{d} r, B]=+\{\mathrm{d} / \mathrm{d} r, E\}
$$

and

$$
[\mathrm{d} / \mathrm{d} r, E]=-\{\mathrm{d} / \mathrm{d} r, B\}
$$

This leads to slightly modified set of Maxwell Electromagnetic Field Equations:

$$
\begin{gathered}
\nabla \times \boldsymbol{B}=\frac{1}{c} \frac{\partial \boldsymbol{E}}{\partial t}+\nabla T \\
\nabla \times \boldsymbol{E}=-\frac{1}{c} \frac{\partial \boldsymbol{B}}{\partial t}
\end{gathered}
$$




$$
\begin{gathered}
\nabla \cdot \boldsymbol{E}=+\frac{1}{c} \frac{\partial T}{\partial t} \\
\nabla \cdot \boldsymbol{B}=0
\end{gathered}
$$

Then following the theoretical ideas as laid out in (for example) "Classical Electricity \& Magnetism" by Abraham \& Becker pages 220-221, it is seen that Equation (11) implies $\boldsymbol{B}=\nabla \times \boldsymbol{A}$ and it then follows that (9) implies $\boldsymbol{E}=-\nabla \phi-\frac{1}{c} \frac{\partial \boldsymbol{A}}{\partial t}$.

When these values are inserted into (8) and (10), those equations become

$$
\nabla \times \nabla \times \boldsymbol{A}+\frac{1}{c^{2}} \frac{\partial^{2} \boldsymbol{A}}{\partial t^{2}}+\frac{1}{c} \nabla \dot{\phi}=\nabla T
$$

and

$$
-\frac{1}{c} \nabla \cdot \dot{\boldsymbol{A}}-\nabla^{2} \phi=\frac{1}{c} \frac{\partial T}{\partial t}
$$

The first of these equations simplifies to

$$
\nabla(\nabla \cdot \boldsymbol{A})-\nabla^{2} \boldsymbol{A}=-\frac{1}{c^{2}} \frac{\partial^{2} \boldsymbol{A}}{\partial t^{2}}-\frac{1}{c} \nabla \dot{\phi}+\nabla T
$$

Then, to quote Abraham \& Becker, (7b) only specifies the curl of the vector $\boldsymbol{A}$. Its sources are still at our disposal and these are defined by laying down the condition

$$
\nabla \cdot \boldsymbol{A}=-\frac{1}{c} \dot{\phi}
$$

Using this in the two preceding equations leads to

$$
\nabla^{2} \phi-\frac{1}{c^{2}} \frac{\partial^{2} \phi}{\partial t^{2}}=-\frac{1}{c} \frac{\partial T}{\partial t}
$$

and

$$
\nabla^{2} \boldsymbol{A}-\frac{1}{c^{2}} \frac{\partial^{2} \boldsymbol{A}}{\partial t^{2}}=-\nabla T
$$

respectively.

\section{Results}

Note that from Equation (6) above

$$
T=-\frac{1}{c} \dot{\phi}+\nabla \cdot \boldsymbol{A}
$$

and, by (12), this gives

$$
T=2 \nabla \cdot \boldsymbol{A}=-\frac{2}{c} \dot{\phi}
$$

so the new scalar field, $T$, which Jack referred to as the "Temporal Field", is not zero when this identification is introduced but remains to make a definite contribution to the theory.

It follows that, if $\boldsymbol{B}=0$, then $\boldsymbol{A}=\nabla S$ and (13) becomes 


$$
\nabla\left(\nabla^{2} S-\frac{1}{c^{2}} \frac{\partial^{2} S}{\partial t^{2}}\right)=-\nabla T
$$

that is

$$
\nabla\left(\nabla^{2} S-\frac{1}{c^{2}} \frac{\partial^{2} S}{\partial t^{2}}+T\right)=0,
$$

which, if $\nabla T=0$, results in the equation for $S$ given in that CIA released article [5].

Note also that from the final equation above it may be deduced that

$$
\nabla^{2} S-\frac{1}{c^{2}} \frac{\partial^{2} S}{\partial t^{2}}+T=0
$$

if it is assumed that the constant of integration is zero. Again this assumption, because assumption it is, is in accordance with the above mentioned article [5].

However, if $T$ is given by Equation (6) and since $A=\nabla S$, it follows that

$$
T=2 \nabla^{2} S
$$

and substituting this into the equation above gives

$$
\nabla^{2} S-\frac{1}{c^{2}} \frac{\partial^{2} S}{\partial t^{2}}+2 \nabla^{2} S=0
$$

or

$$
\nabla^{2} S-\frac{1}{3 c^{2}} \frac{\partial^{2} S}{\partial t^{2}}=0,
$$

which indicates a wave speed of $c \sqrt{3}$; that is, a speed greater than that of light.

It is seen, therefore, that, if the quaternion form of Maxwell's Electromagnetic Field Equations is used, the normal theory follows through as usual, including the more novel notions introduced in the CIA released article mentioned [5]. However, in this case the resulting scalar field turns out to be described by an equation which indicates the wave speed to be greater than that of light; that is the scalar wave is superluminal.

One more factor must now be brought into focus to ascertain the actual wave speed of such a gravitational expression once away from any massive bodies.

\section{Total and Partial Time Derivatives and Their Role in Wave Speeds within an Aetherial Medium}

K. Thornhill [18] (1984) specifies the correct Total Time Derivative to be used in calculations specifying the medium's system dynamics as bound to mass, and so implying a return to a partial time derivative in the medium's unbound stationary state.

From K. Thornhill [18] (1984):

... it is seen that, for general unsteady motion of a gas in three space-variables $x_{i},(i=1,2,3)$ when the fluid velocity components are denoted by $u_{i}$, the governing equations may be written, using the summation convention, 


$$
\begin{gathered}
\text { (Mass) } \frac{D v}{D t}-\frac{v \partial u_{i}}{\partial x_{i}}=-A v^{2} \\
\text { (Momentum) } \frac{D u_{i}}{D t}+\frac{v \partial p}{\partial x_{i}}=B_{i} v \\
\text { (Energy) } \frac{D S}{D t}=v(H-A p v) / T
\end{gathered}
$$

here $p$ denotes pressure, $v$ specific volume, $S$ specific entropy, $T$ absolute temperature and the total time-derivative, moving with the fluid, is given by

$$
\frac{D}{D t}=\frac{\partial}{\partial t}+u_{i} \partial / \partial x_{i}
$$

In the assembly of Maxwell $s$ equations, the time-derivatives which occur in Ampere's rule and in the laws of induction have invariably been interpreted as the partial derivative $\partial / \partial t$. This is not acceptable in the concept of a gas-like ethereal medium, where the ethereal velocity may vary from point to point and with time, and the Newtonian frame of reference may be chosen so that its origin moves at any constant speed, independent of the ethereal motion. To satisfy the requirements of a gas-like ether unambiguously, the time-derivative in Ampere's rule and the laws of induction can only be interpreted as the total time-derivative moving with the ethereal flow, namely $D / D t$, as defined in Equation (14) above.

Once away from mass and the bound condition the return to the partial time derivative in the medium's stationary state then permits near instantaneous longitudinal wave propagation speeds [9] [13]. Wallace Thornhill states: "The crucial difference between the near-infinite speed of the electric force and the relative dawdle of light on any cosmic scale is that the electric force is longitudinal." [19].

\section{Discussion Points and Conclusion}

Adjustment of the electromagnetic axiomatic basis of modern physical theory to include an additional seventh scalar part of the electromagnetic field as that defined by P.M. Jack, could revolutionize electromagnetic and gravitational theory. It appears possible that the mathematics and engineering have already been derived and put to use by the United States Government. If public, academic and nonmilitary industrialized applications of this science were fruitfully applied to serve the general population, clean energies and anti-gravitational effects could become the ordinary province of the next generations. This certainly appears to be a topic deserving of more detailed scientific investigation in the future.

\section{Conflicts of Interest}

The authors declare no conflicts of interest regarding the publication of this paper.

\section{References}

[1] Maxwell, J.C. (2007) A Treatise on Electricity and Magnetism. Merchant Books. 
https://openlibrary.org/publishers/Merchant_Books

[2] CIA Reading Room, 6/29/20.

https://www.cia.gov/library/readingroom/docs/CIA-RDP96-00788R001900680014$\underline{4 . p d f}$

[3] Bearden, T. (1988) AIDS: Biological Warfare. Tesla Book Company, Greenville.

[4] Bearden, T. (1991) Gravitobiology. Cheniere Press, Santa Barbara.

[5] CIA Reading Room, 6/29/20. https://www.cia.gov/library/readingroom/docs/CIA-RDP96-00792R0005002400016.pdf

[6] Jack, P.M. (2003) Physical Space as a Quaternion Structure, 1; Maxwell Equations. A Brief Note.

[7] Jack, P.M. (2006-2007) Three Quaternion Papers. Timefoot Books.

[8] Maxwell, J.C. (1865) A Dynamical Theory of the Electromagnetic Field. (2013 Reprint, Isha Books, New Delhi) https://doi.org/10.5479/sil.423156.39088007130693

[9] Norman, R.L. and Dunning-Davies, J. (2020) Parana Journal of Science and Education, 6, 51-64.

[10] Anastasovski, P.K., Bearden, T.E., et al. (2001) Foundations of Physics Letters, 14, 87-94.

[11] Sweet, F. and Bearden, T.E. Utilizing Scalar Electromagnetics to Tap Vacuum Energy. Proceedings of the 26 th Intersociety Energy Conversion Engineering Conference. https://www.semanticscholar.org/paper/Utilizing-Scalar-Electromagnetics-To-TapVacuum-Sweet-Bearden/9d14594e2c522d6b280cd26d9b85a949ac12c553

[12] Bearden, T. and Bedini, J. (2011) Free Energy Generation. Energetic Productions, Inc. http://www.cheniere.org/

[13] Norman, R. and Dunning-Davies, J. (2018) Hadronic Journal, 41, 1. https://www.researchgate.net/publication/327209709_Probabilistic_Mechanics_the_ Hidden_Variable

[14] Whittaker, E.T. (1904) Proceedings of the London Mathematical Society, Series 2, 1, 367-372. https://doi.org/10.1112/plms/s2-1.1.367

[15] Whittaker, E.T. (1903) Mathematische Annalen, 57, 333-355. https://doi.org/10.1007/BF01444290

[16] Whittaker, E.T. and Watson, G.N. (1935) A Course of Modern Analysis. C.U.P., Cambridge.

[17] Whittaker, E.T. (1931) A History of Theories of Aether and Electricity. Thomas Nelson \& Sons, London.

[18] Thornhill, C.K. (1984) Speculations in Science and Technology, 8, 273-280.

[19] Electric Gravity in an Electric Universe 6/29/20.

https://www.holoscience.com/wp/electric-gravity-in-an-electric-universe 